\title{
Estudo etnobotânico de plantas medicinais utilizadas como hipoglicemiantes por usuários do Programa de Fitoterapia da Universidade Federal do Maranhão, Brasil
}

\begin{abstract}
Ethnobotanical survey of medical plants used as hypoglycemic by users of Phytotherapy
\end{abstract} Program of Federal University of Maranhão - UFMA, Brazil.

\author{
K. A. Santos ${ }^{1 *}$; C. M. Vilanova ${ }^{2}$

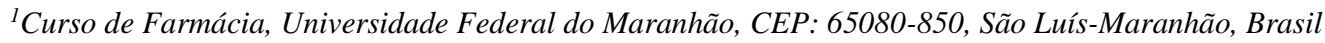 \\ ${ }^{2}$ Departamento de Farmácia, Laboratório de Farmacobotânica, Universidade Federal do Maranhão, CEP: 65080 - \\ 850, São Luís-Maranhão, Brasil \\ *kayquealmeida@yahoo.com.br
}

(Recebido em 11 de agosto de 2016; aceito em 25 de abril de 2017)

\begin{abstract}
O uso de plantas medicinais no tratamento de enfermidades tem ocorrido há muitos anos, sendo a única terapia de certas comunidades. O Diabetes Mellitus é uma desordem metabólica caracterizada pela hiperglicemia, associada a outros fatores de risco. Este trabalho teve como finalidade realizar um levantamento de plantas medicinais utilizadas como hipoglicemiantes por usuários do Programa de Fitoterapia da Universidade Federal do Maranhão - UFMA, São Luís, Maranhão, Brasil. Foram entrevistadas 100 pessoas, através de questionários semiestruturados com obtenção de dados socioeconômicos e variáveis farmacobotânicas com listagem livre de plantas, aplicados entre os meses de março e junho de 2016. A maioria dos entrevistados era do sexo feminino (62\%), com idade entre 30 e 59 anos $(62 \%)$ e ensino médio completo $(48 \%)$. Foram mencionadas 28 espécies vegetais com a finalidade hipoglicêmica, sendo a berinjela (Solanun melongena L.) a mais citada (31\%). As folhas foram as partes da planta mais utilizadas (45\%) e infusão foi a forma de preparo mais indicada pelos entrevistados $(43 \%)$. $\mathrm{O}$ estudo das plantas realizado poderá auxiliar estudos posteriores no desenvolvimento de novos medicamentos para o tratamento de diabetes.

Palavras-chave: Etnofarmacológico. Diabetes. Medicina Popular.
\end{abstract}

The use of medicinal plants in treatment of diseases has been occurred for many years, being the therapy of certain communities. Diabetes Mellitus is a metabolic disorder characterized by hyperglycemia, associated with others risk factors. This work was to carry out a survey of medicinal plants used as hypoglycemic by users of Phytotherapy Program of the Federal University of Maranhão - UFMA, São Luís, Maranhão, Brazil. 100 people were interviewed, through semi-structured questionnaires to obtain socioeconomic data and pharmacobotany variables with free listing of plants, applies between the months of March and June 2016. The majority of respondents were women (62\%), aged between 30 and 59 years (62\%) and completed high school (48\%). 28 plant species were mentioned with purpose hypoglycemic, being the eggplant (Solanun melongena L.) most cited (31\%). The leaves were the plant parts most used $(45 \%)$ and infusion was the way preparation most indicated by respondents $(43 \%)$. The study of plants made may assist further studies on the development of new drugs for the treatment of diabetes.

Keywords: Ethnopharmacological. Diabetes. Popular medicine.

\section{INTRODUÇÃO}

A etnobotânica é a ciência que estuda as relações entre as sociedades humanas com as interações ecológicas, genéticas, evolutivas, simbólicas e culturais com as plantas, além do conhecimento que essas sociedades têm sobre as plantas, como suas classificações e usos de cada uma $[1,2,3]$.

Segundo a Organização Mundial da Saúde (OMS), 80\% da população mundial já fez uso de alguma planta medicinal para tratar alguma patologia ou algum sintoma, reconhecendo em 1978 
o uso de tais plantas no combate de determinadas doenças, além da manutenção da saúde, valorizando o uso das plantas medicinais no que tange ao âmbito sanitário de um país [4]. Desde então, a OMS vem recomendando, desde então, aos órgãos de saúde pública que façam o levantamento etnobotânico, identifiquem as plantas botanicamente e que seja estimulado o seu uso [5].

Tendo isso em vista, os levantamentos etnobotânicos são de fundamental importância para o conhecimento e o estudo de plantas com finalidades medicinais [6], o que contribui não somente para um uso melhor pela população, como também pode expor esse conhecimento para o combate de diversas doenças [7], principalmente no Brasil, onde há uma das maiores biodiversidade do mundo e vários usos de plantas associadas ao conhecimento popular e a maior parte dessa diversidade vegetal ainda está inexplorada e pouco estudada [8,9]. Entretanto, esse conhecimento tem estado em risco por conta da degradação de ambientes naturais, como as florestas tropicais, o que leva à diminuição da disponibilidade de plantas medicinais [10].

Foram várias as ações, no Brasil, de valorização do uso de plantas medicinais e da fitoterapia, sendo a primeira a criação do Programa de Pesquisa de Plantas Medicinais (PPPM) que funcionou pela Central de Medicamentos (CEME) ente os anos de 1982 e 1997 e promovia estudos e pesquisas voltadas aos fins terapêuticos de plantas medicinais utilizadas pela população[11]. A Resolução CIPLAN n ${ }^{\circ} 08$ de 08 de março de 1988 foi a primeira a regularizar a Fitoterapia nos serviços de saúde e nas Unidades Assistenciais Médicas [12].

Em 2006, o Ministério da Saúde do Brasil lançou a Política Nacional de Práticas Integrativas e Complementares (PNPIC) que inclui não somente políticas, com também ações e responsabilidades governamentais na inclusão de práticas, como a Fitoterapia, no Sistema Único de Saúde (SUS), o sistema público de saúde brasileiro, abrindo o mercado dos fitoterápicos no Brasil [11, 13]. Após isso, foi criada a Política Nacional de Plantas Medicinais e Fitoterápicos (PNPMF) que garante o acesso seguro e uso racional de plantas medicinais, o desenvolvimento de tecnologias e inovações e o uso sustentável da biodiversidade brasileira [14].

Mesmo havendo grande pesquisa e estudos na área de plantas medicinais, ainda há poucos projetos e programas devido à pouco investimento na inserção da fitoterapia no SUS. O Ministério da Saúde detectou, em 2004, 116 experiências municipais/estaduais com plantas medicinais e fitoterapia no Brasil, mencionando que essas experiências ocorrem principalmente na atenção básica [13].

Um grande fator para a criação de programas de fitoterapia no Brasil foi o Programa Farmácias Vivas, no estado do Ceará, pelo professor Francisco José de Abreu Matos. Esse programa é o primeiro que se baseou no uso científico de plantas medicinais para realizar a assistência social farmacêutica, produzindo medicamentos fitoterápicos acessíveis à população, sobretudo a mais carente, se tornando referência parra todo o país [15, 16].

No Maranhão, o Programa de Fitoterapia da Universidade Federal do Maranhão (UFMA) tem por objetivo o estudo da flora maranhense e suas propriedades farmacológicas, contribuindo para a ampliação do conhecimento científico na área de produtos naturais, além de promover a melhoria da saúde da comunidade, em especial a de baixa renda [17].

Considerada como uma das maiores desordens endócrinas, a Diabetes Mellitus é uma doença metabólica que altera o metabolismo de carboidratos, lipídeos e proteínas [18]. Não sendo somente uma desordem isolada, mas um grupo de desordens metabólicas, como a hiperglicemia, sede intensa, excreção urinária aumentada, cetonemia e cetonúria [19]. Essa desordem afeta 450 milhões de adultos ao redor do mundo, sendo que uma entre onze pessoas tem diabetes, sendo registrado, no Brasil, mais 130 mil mortes e um gasto de 21.8 bilhões de dólares no tratamento de pessoas com diabetes [20].

Dentro desse contexto, este trabalho tem como finalidade realizar um levantamento etnobotânico das plantas utilizadas como hipoglicemiantes pelos usuários do Programa de Fitoterapia da Universidade Federal do Maranhão em São Luís, Maranhão, a fim de promover o registro, análise e preservação desse saber para estudos posteriores no desenvolvimento de medicamentos para o tratamento de diabetes. 


\section{MATERIAL E MÉTODOS}

O levantamento etnobotânico sobre as plantas medicinais hipoglicemiantes se deu com os usuários do Programa de Fitoterapia, localizado na Cidade Universitário Dom Delgado da Universidade Federal do Maranhão, São Luís, Maranhão, Brasil. O estudo foi de caráter quantitativo, descritivo e exploratório, mediante aplicação de questionários semiestruturados entre os meses de março e junho de 2016.

As variáveis do questionário se baseavam em dados socioeconômicos e demográficos, além de listagem livre de plantas e dados farmacobotânicos, como: as partes do vegetal e modo de uso. Foram convidadas a participar da pesquisa 100 pessoas com idade maior ou igual a 18 anos e que tivessem algum conhecimento sobre plantas medicinais hipoglicemiantes. Os questionários foram aplicados no próprio local do Programa de Fitoterapia, após esclarecimento do objetivo e da metodologia da pesquisa e concordância com assinatura do Termo de Consentimento Livre e Esclarecido (TCLE).

Os critérios de inclusão estabelecidos foram ser usuário do Programa de Fitoterapia da UFMA, ter maioridade, conhecer e/ou usar plantas como hipoglicemiantes e concordar com TCLE. Os critérios de exclusão adotados foram não se enquadrar nos critérios de inclusão e solicitar saída da pesquisa.

Os dados foram analisados através de cálculos percentuais e de frequência relativa pelo software Microsoft Excel ${ }^{\circledR} 2013$ e dispostos em gráficos e tabelas. A pesquisa foi aprovada pelo Comitê de Ética em Pesquisa (CEP) da Universidade Federal do Maranhão (UFMA) sob o parecer $\mathrm{n}^{\mathrm{o}} 154.698$.

\section{RESULTADOS E DISCUSSÃO}

Após análise dos 100 questionários observou-se que a maioria dos entrevistados é do gênero feminino (62\%), está na faixa de 30-59 anos (62\%), é de origem urbana (72\%), usa celular e internet como principal meio de comunicação (52\%), tem o ensino médio completo (39\%) e não informou sua renda salarial (43\%), tendo a renda de 1 a 3 salários mínimos como a segunda renda mais citada, segundo a Tabela 1 .

As mulheres são as grandes detentoras do conhecimento acerca de plantas medicinais e são as que mais participam do processo de transmissão desse conhecimento [21], além do fato delas dominarem mais o conhecimento das plantas que crescem nas proximidades de suas residências, ao contrário dos homens que possuem um maior conhecimento acerca das plantas que crescem em matos [22]. Como os usuários entrevistados geralmente eram os que estavam para se consultar, a grande presença de mulheres em relação aos homens é justificada também pelo fato das mulheres demonstrarem um maior cuidado com a saúde do que os homens [23].

Os usuários entrevistados são bem mais representados por adultos (62\%), seguido por idosos (23\%) e por adultos jovens (15\%). O conhecimento e uso de plantas medicinais quase sempre é encontrado em pessoas adultas ou idosas, já que eles possuem uma maior experiência quanto ao uso de plantas, além de possuírem um maior legado e confiança em tal terapêutica [24]. Além disso, pessoas a partir de 30 anos possuem uma maior aderência a participar de pesquisas e entrevistas [25]. Como o Programa de Fitoterapia se encontra em um Campus Universitário, onde há a presença de pessoas que acabaram de completar o Ensino Médio ou já são formadas, a prevalência de pessoas nessa faixa de escolaridade é justificada por esse motivo.

O grau de escolaridade tem um grande papel de influência no uso, devido ao fato de muitas pessoas ainda acreditarem que plantas medicinais serem isentas de efeitos tóxicos e colaterais, contradizendo estudos científicos que comprovam o contrário [24]. Isso se deve à crença das plantas medicinais serem mais seguras que os medicamentos industrializados, pois são de fontes naturais e não passam por todo o processamento industrial. 
Tabela 1: Distribuição numérica e percentual das variáveis socioeconômicas e demográfica dos usuários do Programa de Fitoterapia da Cidade Universitária Dom Delgado, UFMA, São Luís - MA, Brasil,

\begin{tabular}{|c|c|c|}
\hline Variáveis & $\mathbf{N}$ & $\%$ \\
\hline \multicolumn{3}{|l|}{ Sexo } \\
\hline Masculino & 38 & 38 \\
\hline Feminino & 62 & 62 \\
\hline \multicolumn{3}{|l|}{ Faixa etária } \\
\hline 18-29 anos & 15 & 15 \\
\hline $30-59$ anos & 62 & 62 \\
\hline$\geq 60$ anos & 23 & 23 \\
\hline \multicolumn{3}{|l|}{ Renda salarial } \\
\hline Até 1 salário mínimo & 12 & 12 \\
\hline $1^{\text {a }} 3$ salários mínimos & 33 & 33 \\
\hline Mais de 3 salários & 12 & 12 \\
\hline Não informado & 43 & 43 \\
\hline \multicolumn{3}{|l|}{ Meios de comunicação } \\
\hline TV & 31 & 31 \\
\hline Celular/Internet & 52 & 52 \\
\hline Rádio & 17 & 17 \\
\hline Revistas & 1 & 1 \\
\hline \multicolumn{3}{|l|}{ Escolaridade } \\
\hline Sem escolaridade & 4 & 4 \\
\hline Alfabetizado & 12 & 12 \\
\hline Ensino fundamental & 14 & 14 \\
\hline Ensino médio & 48 & 48 \\
\hline Ensino superior & 22 & 22 \\
\hline Total & 100 & 100 \\
\hline
\end{tabular}

Embora a televisão seja o meio de comunicação mais utilizado na população brasileira [25], o aumento da utilização da Internet, principalmente em conjunto com o Celular, tem se tornado uma grande fonte de obtenção de informações, além de ser um meio de comunicação prático e um recurso de entretenimento [24].

Observa-se pela Figura 1 que a grande maioria desse conhecimento sobre plantas medicinais veio dos pais (48\%) e avós (14\%), devido a transmissão oral de conhecimento entre gerações e que $82 \%$ dos usuários do programa afirmaram que tem o costume de fazer o uso de plantas medicinais. Isso corrobora com estudos anteriores [26], onde todos os entrevistados afirmaram que haviam obtido o conhecimento de plantas medicinais através de familiares.

A

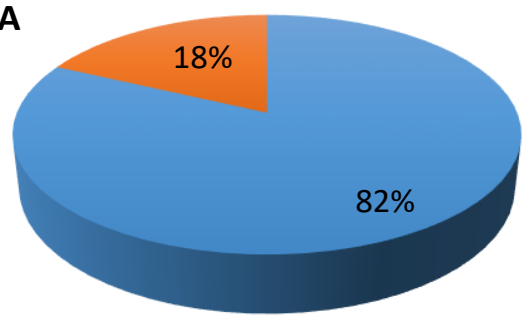

$\square \operatorname{Sim} \square$ Não
B

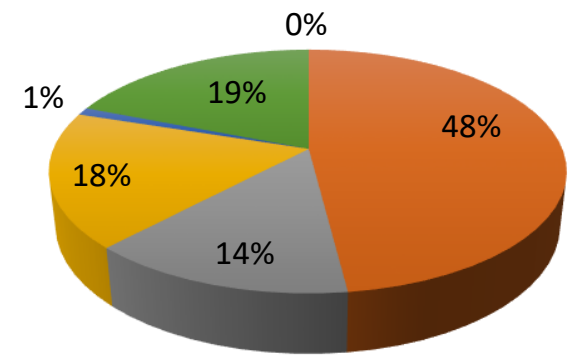

Pais $\square$ Avós $\square$ Amigos $\square$ Cônjugue $\square$ Outros

Figura 1 - (A) Distribuição percentual de pessoas que tem o costume de fazer uso de plantas medicinais $e(B)$ obtenção do conhecimento da indicação de plantas medicinais para uso hipoglicemiante pelos usuários do Programa de Fitoterapia da Cidade Universitária Dom Delgado, UFMA, São Luís - MA, Brasil, 2016. 
Quanto ao conhecimento ou uso de plantas medicinais como hipoglicemiantes, observou-se que 58\% dos entrevistados tinham algum conhecimento sobre essas plantas, como verificado na Figura 2. Muitos deles, adquiridos através da tradição e pela influência de outras pessoas, como visto na Figura 1B. A influência dos meios de comunicação podem reforçar o uso de plantas medicinais.

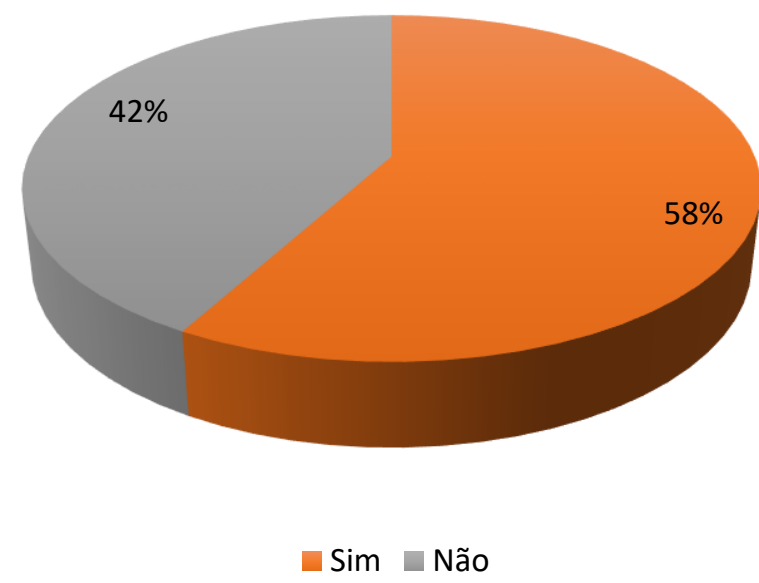

Figura 2 - Distribuição percentual de pessoas que conhecem ou já usaram plantas medicinais hipoglicêmicas que apresenta ou não o diabetes no Programa de Fitoterapia da Cidade Universitária Dom Delgado, UFMA, São Luís - MA, Brasil, 2016.

$\mathrm{Na}$ Tabela 2, observa-se que foram citadas 28 espécies vegetais utilizadas com o fim terapêutico de hipoglicemia. As plantas mais citadas pelos entrevistados foram a berinjela (Solanun melongena L.) com 31\%; a insulina vegetal (Cissus sicyoides L.), com 12\%; o quiabo (Abelmoschus esculentus L. Moench), com 10\%; e o jambolão (Syzygium cumini (L.) Skeels), com $6 \%$.

Um levantamento de plantas medicinais realizado no bairro do Maracanã, em São Luís, Maranhão, mostrou 27 espécies vegetais com a finalidade hipoglicêmica, sendo que 10 dessas também foram citadas neste trabalho, dentre as quais podemos destacar pau-ferro, berinjela, pautenente, insulina vegetal e o jambolão, inclusive a parte utilizada e preparo são similares [24].

A berinjela apresenta vários estudos pré-clínicos mostrando seu potencial no combate da Diabetes. Derivi et al. (2002) [27] mostraram que a casca da berinjela possui um composto responsável pelo efeito hipoglicêmico, ao submeterem um grupo de ratos adultos normais e diabéticos induzidos por aloxana que receberam rações à base de caseína, berinjela com casca e sem casca, e de casca de berinjela por um tempo de 42 dias. Qonita et al. (2013) [28] mostraram que o extrato da berinjela possui um efeito antihiperglicêmico, ao ser administrado nas doses entre 383 a $1372 \mathrm{mg} / \mathrm{Kg}$ durante 15 dias.

A insulina vegetal também é muito utilizada pela população na finalidade de diminuir a glicemia, o que coincide com dados da literatura. Santos et al. (2008) [29] realizou um estudo clínico de fase II com a infusão das folhas da planta em pacientes intolerantes à glicose e diabéticos em um hospital. $\mathrm{O}$ resultado mostrou que a infusão teve ação hipoglicemiante com os pacientes, embora não tenha sido de forma significativa nos pacientes diabéticos. Ainda há mais estudos da insulina vegetal no combate da diabetes [30,31].

As propriedades antioxidantes do quiabo [32] pode ser um grande fator pelo seu potencial antidiabético e antihiperlipidêmico, como demonstrado em investigações in vivo em animais diabéticos induzidos por estreptozotocina, onde tais animais receberam o pó das cascas e das sementes, resultando na diminuição dos níveis de glicemia, aumento do peso corporal, aumento 
da proteína total, diminuição da hemoglobina glicada (HbA1c) e retorno dos níveis elevados do perfil lipídico aos níveis normais [33].

Há estudos in vivo que comprovam o efeito hipoglicemiante do Jambolão, não somente em extratos alcoólicos e aquosos das sementes [34, 35, 36], como em extratos aquosos de folhas [37, 38,39 ]. Estudos in vitro tem mostrado que o extrato metanólico de Jambolão é capaz de aumentar a expressão do transportador de glicose (GLUT-4), do receptor ativado por proliferadores de peroxissoma gama (PPAR $\gamma$ ) e da fosfatidil-inositol 3-quinase (PI 3-quinase), resultando no aumento da captação de glicose pelas células musculares [40].

Outras espécies vegetais menos citadas pelos usuários, como o Aloe vera L. (babosa), Bauhinia forticata Link (pata de vaca), Quassia amara L. (pau tenente), Annona muricata L. (graviola), Azadirachta indica A. Juss. (nim) merecem ser relatadas aqui por conta de estudos [41, 42, 43, 44, 45] mostrarem suas propriedades hipoglicêmicas.

São vários os mecanismos de ação de plantas hipoglicemiantes, como a estimulação da secreção de insulina das células beta das ilhotas pancreáticas ou a inibição da degradação de insulina [46]; adrenomimetismo, bloqueio do canal de potássio na célula beta pancreática [47]; redução da resistência à insulina [46]; regeneração e reparo das células beta pancreáticas [48]; aumento do número de células nas ilhotas pancreáticas [48]; estimulação da secreção de insulina [47]; estimulação da glicogêneses e glicólise hepática [50]; efeito protetor na destruição de células beta pancreáticas [51]; diminuição da atividade do cortisol [52]; inibição da alfa-amilase [53]; prevenção do estresse oxidativo que está relacionado com a disfunção da célula beta pancreática [54].

Foram identificadas várias partes da planta medicinal usadas para o preparo (Figura 3), sendo as folhas as mais usadas (45\%), seguidas pelos frutos (21\%), raízes (17\%), cascas do tronco (14\%) e sementes (3\%). Esses dados são comprovados com estudos que mostram as folhas como a parte da planta, mais utilizada nas preparações caseiras [26, 55], além do fato delas serem mais fáceis para a coleta, devido à sua maior exposição e maior concentração de princípios ativos na maioria das espécies vegetais [56].

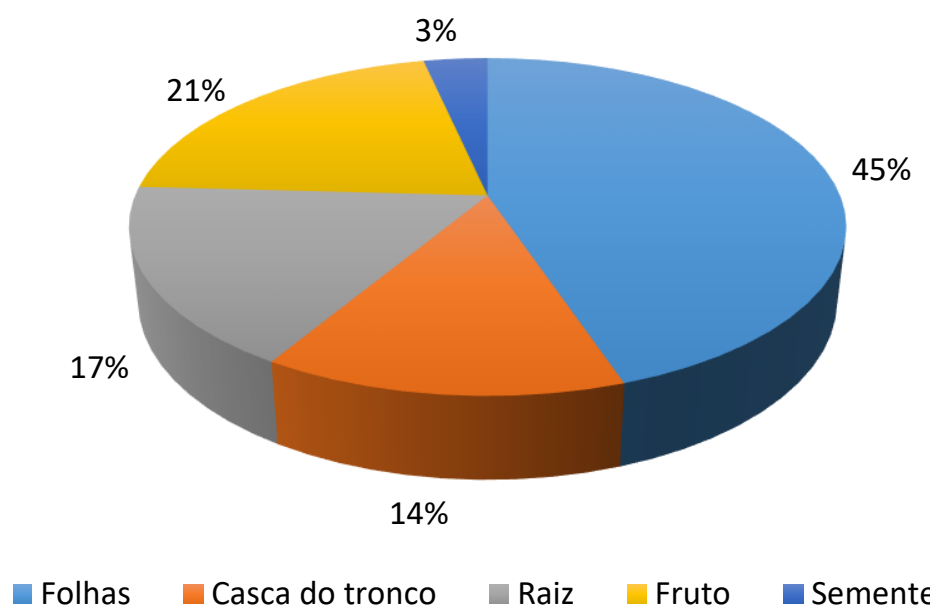

Figura 3 - Distribuição percentual da parte usada da planta medicinal hipoglicemiante, segundo os usuários do Programa de Fitoterapia da Cidade Universitária Dom Delgado, UFMA, São Luís - MA,

Brasil, 2016.

Quanto ao modo de preparo, a Figura 4 revela que a forma conhecida como infusão é a mais citada (43\%), seguida pela decocção (20\%), maceração (17\%), in natura $(17 \%)$ e suco $(3 \%)$. Esses dados mostram similaridade com alguns estudos, que mostram a infusão e a decocção como as preparações caseiras de plantas medicinais mais utilizadas [26, 57]. 
Tabela 2: Relação de plantas utilizadas como hipoglicemiantes, parte utilizada e modo de preparo mencionados por usuários do Programa de Fitoterapia da Universidade Federal do Maranhão - UFMA, São Luís-MA, Brasil, 2016

\begin{tabular}{|c|c|c|c|c|c|}
\hline Nome popular & Nome científico & Família & Parte utilizada & Preparo & $\%(\mathrm{~N})$ \\
\hline Açoita cavalo & Luehea grandiflora Mart. Et. Zucc & Tiliaceae & Folha & Infusão & $2 \%(2)$ \\
\hline Amora & Morus nigra $\mathrm{L}$. & Moraceae & Folhas & Infusão & $1 \%(1)$ \\
\hline Babosa & Aloe vera $\mathrm{L}$ & Asphodelaceae & Gel das folhas & Infusão & $1 \%(1)$ \\
\hline Barbatimão & Stryphnodendron adstringens Mart. & Fabaceae & Casca do tronco & Decocção & $1 \%(1)$ \\
\hline Batata doce & Ipomoea batatas (L.) Lam. & Convolvulaceae & Raiz & In natura & $1 \%(1)$ \\
\hline Berinjela & Solanun melongena $\mathrm{L}$. & Solanaceae & Fruto & In natura & $31 \%(26)$ \\
\hline Canela & Cinnamomum zeylanicum Breyn. & Lauraceae & Casca do tronco & Infusão & $1 \%(1)$ \\
\hline Cebola roxa & Allium cepa $\mathrm{L}$. & Lilaceae & Bulbo & Decocção & $1 \%(1)$ \\
\hline Cipó de Jabuti & Bauhinia rutilans Spru. Ex Benth & Caesalpinioideae & Folhas & Maceração & $1 \%(1)$ \\
\hline Erva mate & Ilex paraguariensis St. Hill & Aquifoliaceae & Folhas & Infusão; Decocção & $1 \%(1)$ \\
\hline Gengibre & Zingiber officinale Roscoe & Zingiberaceae & Raiz & Infusão & $1 \%(1)$ \\
\hline Graviola & Annona muricata $\mathrm{L}$. & Annonaceae & Fruto & In natura & $1 \%(1)$ \\
\hline Guajuru & Chrysobalanus icaco (L.) L. & Chrysobalanaceae & Raiz & Decocção & $1 \%(1)$ \\
\hline Insulina Vegetal & Cissus sicyoides $\mathrm{L}$. & Vitaceae & Folhas & Infusão & $12 \%(10)$ \\
\hline Jambolão & Syzygium cumini (L.) Skeels & Myrtaceae & Folhas; Casca do tronco & Maceração & $6 \%(5)$ \\
\hline Juçara & Euterpe edulis Mart. & Arecaceae & Raiz & Decocção & $1 \%(1)$ \\
\hline Laranja da terra & Citrus aurantium L. & Rutaceae & Folhas e frutos & Suco & $1 \%(1)$ \\
\hline Língua de vaca & Chaptalia nutans (L.) Polak & Asteraceae & Folhas & Infusão & $1 \%(1)$ \\
\hline Linhaça & Linum usitatissimun L. & Linaceae & Semente & In natura & $1 \%(1)$ \\
\hline Maxixe & Cucumis anguria $\mathrm{L}$. & Cucurbitaceae & Fruto & Maceração & $2 \%(2)$ \\
\hline Murici & Byrsonima crassa Nied & Malpighiaceae & Casca do tronco & Decocção & $1 \%(1)$ \\
\hline Nin & Azadirachta indica A. Juss. & Meliaceae & Folhas & Infusão & $1 \%(1)$ \\
\hline Noni & Morinda citrifolia L. & Rubiaceae & Fruto & Maceração & $1 \%(1)$ \\
\hline Pata de vaca & Bauhinia forticata Link & Fabaceae & Folhas & Infusão & $6 \%(5)$ \\
\hline
\end{tabular}




\begin{tabular}{|c|c|c|c|c|c|}
\hline Pau Ferro & Caesalpinia ferrea Mart. Ex Tul. & Fabaceae & Folha; Fruto & Infusão; In natura & $1 \%(1)$ \\
\hline Pau tenente & Quassia amara L. & Simaroubaceae & Folha & Infusão & $2 \%(2)$ \\
\hline Quiabo & Abelmoschus esculentus L. Moench & Malvaceae & Goma do fruto & Maceração & $10 \%(8)$ \\
\hline Tentem & Struthantus flexicaulis (Mart.) Mart. & Loranthaceae & Raiz & Infusão & $5 \%(4)$ \\
\hline TOTAL & & & & & $100 \%(83)$ \\
\hline
\end{tabular}




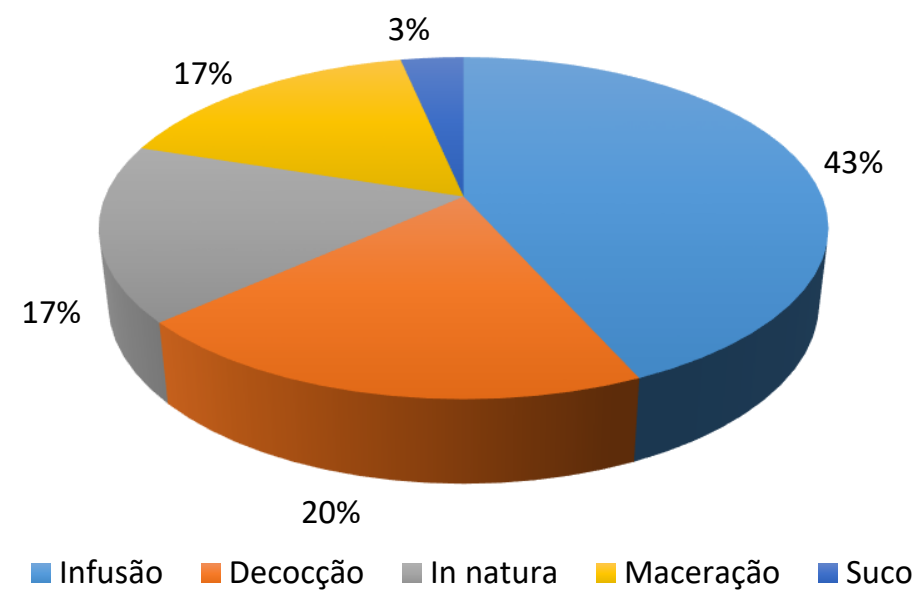

Figura 4 - Distribuição percentual do preparo da planta medicinal hipoglicemiante, segundo os usuários do Programa de Fitoterapia da Cidade Universitária Dom Delgado, UFMA, São Luís - MA, Brasil, 2016.

Como são poucos os estudos clínicos com plantas medicinais, deve-se considerar que algumas plantas podem ter efeitos tóxicos, como hepatotoxicidade, mostrando, portanto, efeito contrário efeito terapêutico desejado [24]. O método de preparação, a parte da planta usada e a via de administração ainda são fatores que podem influenciar a toxicidade [58].

Além do mais, plantas medicinais possuem várias substâncias, em complexas misturas, com pouco conhecimento sobre seus efeitos no organismo, assim como sua toxicidade e efeitos colaterais, bem como seus produtos de biotransformação, que podem causar quadros clínicos severos, quando seus efeitos se instalam a longo prazo [24].

\section{CONCLUSÃO}

A partir dos resultados deste trabalho, percebe-se a importância não somente da realização dos estudos etnobotânicos, que ajudam a compreender o uso e aplicabilidade na terapêutica de determinadas patologias das plantas, como também do conhecimento popular e sua transmissão para as gerações futuras, mostrando que essa sabedoria popular ainda é um dos principais veículos de conhecimento para o tratamento de doenças.

Foi observado que há diversas plantas medicinais utilizadas para o tratamento da Diabetes Mellitus devido à sua ação hipoglicêmica, a qual tem sido registrada e comprovada em trabalhos, corroborando com o conhecimento tradicional popular. A forma de preparo das plantas e parte utilizada para o mesmo as torna ainda mais acessíveis à população, levando-as a serem mais uma alternativa natural para o combate da doença em questão.

Embora essas plantas possuam uma certa efetividade para essa finalidade terapêutica, a qual é comprovada pelo conhecimento científico, é necessário que sejam realizados mais estudos sobre sua segurança e toxicidade, além de esclarecimentos de profissionais capacitados sobre seu uso, preparo e finalidade, a fim de evitar que problemas, como intoxicação, possam ocorrer e que os resultados sejam positivos.

Assim, este trabalho reflete a partir de seus resultados que o conhecimento da população para tratar doenças com plantas continua sendo atual e uma prática ainda bastante difundida, comprovada pelos entrevistados, além de ser uma forma de tratamento bem mais barata $\mathrm{e}$ economicamente viável.

\section{AGRADECIMENTOS}

Ao laboratório de Farmacobotânica do Departamento de Farmácia da Universidade Federal do Maranhão (UFMA), ao Programa de Fitoterapia da UFMA e a Fundação de Amparo à Pesquisa 
e ao Desenvolvimento Científico e Tecnológico do Maranhão (FAPEMA) pelo apoio dado ao projeto.

\section{REFERÊNCIAS BIBLIOGRÁFICAS}

1. Soares MAA, Braga JRP, Mourão AEB, Parente KMS, Filho EGP. Levantamento Etnobotânico das plantas medicinais utilizadas pela população do município de Gurinhém - Paraíba. Revista Homem, Espaço e Tempo. 2009;2(1):36-47.

2. Büttow MV. Etnobotânica e caracterização molecular de Butia sp. 2008. 64f. Dissertação (Mestrado em Fitomelhoramento) - Universidade Federal de Pelotas, Pelotas, 2008.

3. Oliveira, G. L. Etnobotânica nordestina: plantas medicinais da comunidade Muribeca (Jaboatão dos Guararapes - PE, Brasil). 2007. 72f. Dissertação (Mestrado em Biologia Vegetal) - Universidade Federal do Pernambuco, Jaboatão dos Guararapes, 2007.

4. Brasil. Ministério da Saúde. Secretaria de Ciência, Tecnologia e Insumos Estratégicos. Departamento de Assistência Farmacêutica. A Fitoterapia no SUS e o Programa de Pesquisas de Plantas Medicinais da Central de Medicamentos. Brasília, 2006a.

5. Brasil. Ministério da Saúde. Secretaria de Ciência, tecnologia e Insumos Estratégicos. Departamento de Assistência Farmacêutica. Política Nacional de Plantas medicinais e fitoterápicos. Brasília, $2006 \mathrm{~b}$.

6. Ming LC. Coleta de Plantas medicinais. In: Distasi L.C. Plantas medicinais: Arte e Ciência - um guia de estudo interdisciplinar. São Paulo: Nobel, 1999, p.69-86.

7. Amorozo MCM, Gély A. Uso de plantas medicinais por caboclos do baixo Amazonas, Barbacena, PA, Brasil. Bol Museu Paraense Emílio Goeldi, Série Botânica 1998;4(1):47-131.

8. Santos EB, Dantas GS, Santos HB, Diniz MFFM, Sampaio FC. Estudo etnobotânico de plantas medicinais para problemas bucais no município de João Pessoa, Brasil. Rev Bras Farmacog; 2009;19(1B):321-324, doi 10.1590/s0102-695x2009000200024.

9. Alonso JR. Tratado de Fitomedicina: bases clínicas y farmacológicas. Buenos Aires: Isis Ediciones SRL, 1998, 1038 p.

10. Bermúdez A, Oliveira-Miranda MA, Velázquez, D. La investigación etnobotánica sobre plantas medicinales: Una revisión de sus objetivos y enfoques actuales. Interciencia; 2005;30(8):453-459.

11. Maranhão DG. Análise situacional de seis programas de fitoterapia brasileiros [ TCC - Especialização]. Rio de Janeiro: Instituto de Tecnologia em Fármacos/Farmanguinhos, Fundação Oswaldo Cruz; 2011. $49 \mathrm{p}$.

12. BRASIL Ministério da Saúde. Resolução CIPLAN nº. 08, de 08 de março de 1988. Implanta a prática da fitoterapia nos serviços de saúde. Diário Oficial [da] República Federativa do Brasil. Brasília, DF, mar. 1988.

13. BRASIL. Ministério da Saúde. Secretaria de Atenção à Saúde. Departamento de Atenção Básica. Política Nacional de Práticas Integrativas e Complementares no SUS - PNPIC no Sistema Único de Saúde. Brasília: Ministério da Saúde, 2006. 92 p.

14. BRASIL. Ministério da Saúde. Secretaria de Ciência, Tecnologia e Insumos Estratégicos. Departamento de Assistência Farmacêutica e Insumos Estratégicos. Programa Nacional de Plantas Medicinais e Fitoterápicos. Brasília, 2009.

15. Matos FJA. Farmácias Vivas. Fortaleza: Editora da EFC, 1998.

16. Malta JRA, Diniz MFFM, Oliveira RAG. Das plantas medicinais aos fitoterápicos: Abordagem multidisciplinar. João pessoa: PET-FARMÁCIA/CAPES/UFPB, 1999.

17. Demarchi C. A ciência milenar de Terezinha Rêgo. SESC São Paulo. Disponível em: < http://www.sescsp.org.br/online/artigo/4709_A+CIENCIA+MILENAR+DE+TEREZINHA+REGO\#/t agcloud=lista>. Acesso em: 18 mai 2016.

18.Pareek H, Sharma S, Khajja BS, Jain K, Jain GC. Evaluation of hypoglycemic and anti hyperglycemic potential of Tridax procumbens (Linn.). BMC Complement Altern Med 2009; 9:48, doi 10.1186/14726882-9-48.

19. Patel DK, Kumar R, Laloo D, Hemalatha S. Diabetes mellitus: an overview on its pharmacological aspects and reported medical plants having antidiabetic activity. Asian Pac J Trop Biomed; 2012; 2(5):411-20, doi 10.1016/S2221-1691(12)60067-7.

20. International Diabetes Federation, 2011. Unite for diabetes. Bruxelas. Disponível em: <http://www.worlddiabetesday.org/the-campaign/unite-for-diabetes>. Acesso em: 14.06.16.

21. Rodrigues AG, Casali VWD. Plantas medicinais, conhecimento popular e etnociência. In: Rodrigues AG, Andrade FMC, Coelho FMG et al. Plantas Medicinais e Aromáticas: etnoecologia e etnofarmacologia. Viçosa: UFV, 2002. p. 25-76. 
22. Borba AM, Macedo M. Plantas medicinais usadas para a saúde bucal pela comunidade do bairro Santa Cruz, Chapada dos Guimarães, MT, Brasil. Acta Bot. Bras. 2006;20(4):771-782, doi 10.1590/s010233062006000400003 .

23. Costa-Júnior FM, Maia ACB. Concepções de homens hospitalizados sobre a relação entre gênero e saúde. Psicologia: teoria e pesquisa. 2009; 25(1): 055-063, doi 10.1590/s0102-37722009000100007.

24. Gomes PRM, Firmo WCA, Vilanova CM. Estudo etnobotânico de plantas medicinais hipoglicemiantes no bairro Maracanã no município de São Luís, Maranhão, Brasil. Scientia Plena. 2014;10(9)

25.Fuck SB, Athanázio JC, Lima CB, Ming LC. Plantas medicinais utilizadas na medicina popular por moradores da área urbana de Bandeirantes, PR, Brasil. Semina: Ciênc Agrár. 2005;26(3): 291-296, doi $10.5433 / 1679-0359.2005 \mathrm{v} 26 \mathrm{n} 3 \mathrm{p} 291$.

26. Santos JFL, Amorozo MCM, Ming LC. Uso popular de plantas medicinais na comunidade rural de Vargem Grande, município de Natividade da Serra, SP. Rev. Bras. Pl. Med. 2008;10(3):67-81.

27.Derivi SCN, Mendez MHM, Franciscone AD, Silva CS; Castro AF; Luz DP. Efeito hipoglicêmico de rações à base de berinkela (Solanum melongena L.) em ratos. Ciênc Tecnol Aliment. 2002;22(2):164169, doi 10.1590/s0101-20612002000200011.

28.Qonita NR, Zulhaidan M, Sudiarto; Tjahjono HA. The effect of eggplant (Solanum melongena L.) extract peroal against blood glucose level of white rat (Ratus novergicus) wistar strain diabetic model. Int J Pediatr Endocrinol. 2013; Suppl 1:O33, doi 10.1186/1687-9856-2013-s1-o33, doi 10.1186/16879856-2013-s1-033.

29. Santos HB, Modesto-Filho J, Diniz MFFM, Vasconcelos THC, Pereira FSB, Ramalho JA, Dantas JG, Santos EB. Avaliação do efeito hipoglicemiante de Cissus sicyoides em estudos clínicos fase II. Rev Bras Farmacogn. 2008;18(2):70-76, doi 10.1590/s0102-695x2008000100014.

30. Vasconcelos THC, Modesto-Filho J, Diniz MFFM, Santos HB, Aguiar FB, Moreira PVL. Estudo toxicológico pré-clínico agudo com o extrato hidroalcoólico das folhas de Cissus sicyoides L. (Vitaceae). Rev Bras Farmacogn. 2007;17(4):583-591, doi 10.1590/s0102-695x2007000400018.

31. Barbosa WLR, Santos WRA, Pinto LN, Tavares ICC. Flavonóides de Cissus Verticillata e a atividade hipoglicemiante do chá de suas folhas. Rev Bras Farmacogn. 2002;12:13-15, doi 10.1590/s0102$695 \times 2002000300007$.

32. Sabitha V, Ramachandran S, Naveen KR, Panneerselvam K. Investigation of in vivo antioxidant property of Abelmoschus esculentus (L.) Moench. fruit seed and peel powders in streptozotocin-induced diabetic rats. Journal of Ayurveda Integrative Medicine. 2012; 3(4):188-193, doi 10.4103/09759476.104432.

33. Sabitha V, Ramachandran S, Naveen KR, Panneerselvam K. Antidiabetic and antihyperlipidemic potencial of Abelmoschus esculentus (L.) Moench. in streptozotocin-induced diabetic rats. J Pharm Bioallied Sci. 2011;3(3):397-402, doi 10.4103/0975-7406.84447.

34. Mahapatra PK et al. Preliminary studies on glycemic effect of Syzigium cumini seeds. IRCS Medical Science Biochemistry. 1985;3(7):631-632.

35. Indira G, Mohan Ram M, Jamun. In: Fruits. National Institute of Nutrition, Indian Council of Medical Research, Hyderabad, India; 1992, p. 34-37.

36. Prince PSM, Kamalakkannan N, Menon VP. Antidiabetic and antihyperlipidaemic effect of alcoholic Syzigium cumini seeds in alloxan induced diabetic albino rats. J Ethnopharmacol. 2004; 91(2-3): 20921, doi 10.1016/j.jep.2003.11.001.

37. Coimbra TC et al. Plants employed in the treatment of diabetes mellitus results of an ethnopharmacological survey in Porto Alegre, Brazil. Fitoterapia. 1992;63(4):320-322.

38. Teixeira CC et al. The effect of Syzygium cumini (L) skeels on post-prandial blood glucose levels in non-diabetic rats and rats with streptozotocin-induced diabetes mellitus. Journal of Ethnopharmacology. 1997;56(3):209-213, doi 10.1016/s0378-8741(97)01532-8.

39. Soares JCM, Costa ST, Cecim M. Níveis glicêmicos e de colesterol em ratos com Diabetes Mellitus aloxano induzido, tratados com infusão de Bauhinia candicans ou Syzygium Jambolanum. Ciência Rural. 2000;30(1):113-118, doi 10.1590/s0103-84782000000100018

40. Anandharajan R et al. In vitro glucose uptake activity of Aegles marmelos and Syzygium cumini by activation of Glut-4, PI3 kinase and PPAR $\gamma$ in L6 myotubes. Phytomedicine. 2006;13(6):434-441, doi 10.1016/j.phymed.2005.03.008.

41. Rafasekaran S, Sivagnanam K, Subramanian S. Antioxidant effect of Aloe vera gel extract in streptozotocin-induced diabetes in rats. Pharmacological Reports. 2005;57:90-96.

42. Lino CS, Diógenes JP, Pereira BA, Faria RA, Andrade Neto M, Alves RS, Queiroz MG, Sousa FC, Viana GS. Antidiabetic activity of Bauhinia forficata extracts in alloxan-diabetic rats. Biol. Pharm. Bull, 2004;27(1):125-127, doi 10.1248/bpb.27.125. 
43. Husain GM, Singh PN, Singh RK, Kumar V. Antiabetic activity of Standardized Extract of Quassia amara in Nicotinamide-Spreptozotocin-induced diabetic rats. Phytotherapy research. 2011;25(12):1806-1812, doi 10.1002/ptr.3491.

44. Adewole SO, Ojewole AO. Protective effects of Annona muricata Linn. (Annonaceae) leaf aqueous extract on sérum lipid profiles and oxidative stress in hepatocytes of Streptozotocin-Treated diabetic rats. Afri. J. Trad. 2009; 6(1):30-41, doi 10.4314/ajtcam.v6i1.57071.

45. Kazeem MI, Dansu TV, Adeola SA. Inhibitory effect of Azadirachta indica A juss leaf extract on the activities of alpha-amylase and alpha-glucosidase. Pak J Biol Sci. 2013;16(21):1358-62, doi 10.3923/pjbs.2013.1358.1362.

46. Pulok KM, Kuntal M, Kakali M, Peter JH. Leads from Indian medicinal plants with hypoglycemic potentials. J Ethnopharmacol 2006;106:1-28, doi 10.1016/j.jep.2006.03.021.

47. Marles RJ, Farnsworth N. Antidiabetic Plants and their Active Constituents: An update. Prot J Bot Med 1996;1:85-135.

48. Mohamed B, Abderrahim Z, Hassane M, Abdelhafid T, Abdelkhaleq L. Medicinal plants with potential antidiabetic activity - A review of ten years of herbal medicine research (1990-2000). Int J Diabetes Metabol 2006;14:1-25.

49. Esmaeili MA, Yazdanparast R. Hypoglycaemic effect of Teucrium polium: studies with rat pancreatic islets. J Ethnopharmacol 2004;95:27-30, doi 10.1016/j.jep.2004.06.023.

50. Miura T, Itoh C, Iwamoto N, Aato M, Kawai M, Park SR, Suziki I. Hypoglycemic activity of the fruit of the Momordica charantia in Type 2 diabetic mice. J Nutr Sci Vitaminol (Tokyo). 2001;47:340-4, doi 10.3177/jnsv.47.340.

51. Kim MJ, Ryu GR, Chung JS, Sim SS, Min DS, Rhie DJ, Yoon SH, Hahn SJ, Kim MS, Jo YH. Protective effects of epicatechin against the toxic effects of streptozocin on rat pancreatic islets: in vivo and in vitro. Pancreas 2003;26:292-9, doi 10.1097/00006676-200304000-00014.

52. Gholap S, Kar A. Hypoglycaemic effects of some plant extracts are possibly mediated through inhibition in corticosteroid concentration. Pharmazie 2004;59:876-8.

53. Heidari R, Zareae S, Heidarizadeh M. Extraction, Purification, and Inhibitory Effect of Alpha-Amylase Inhibitor from Wheat (Triticum aestivum Var. Zarrin). Pakistan J Nutr 2005;4:101-5, doi 10.3923/pjn.2005.101.105.

54. Hideaki K, Taka-aki M, Yoshihisa N, Dan K, Munehide M, Yoshimitsu Y. Oxidative Stress and the JNK Pathway in Diabetes. Curr Diab Rev 2005;1:65-72, doi 10.2174/1573399052952613.

55. Coelho MFB et al. Medicinal plants in the neighborhood of Serra do Brigadeiro State Park, Minas Gerais State, Brazil. Acta Hortic. 2002, 569(1): 73-77, doi 10.17660/actahortic.2002.569.11.

56. Ming LC; Amaral Júnior, A. Aspectos etnobotânicos de plantas medicinais na reserva extrativista "Chico Mendes". The New York Botanical Garden. 2005. Disponível em <http://www.nybg.org/bsci/acre/www1/medicinal.html>. Acesso em 25 de agosto de 2015.

57. Pinto EPP, Amoroso MCM, Furlan A. Conhecimento popular sobre plantas medicinais em comunidades rurais de mata atlântica-Itacaré, BA, Brasil. Acta Bot. Bras. 2006;20(4):751-762, doi 10.1590/s010233062006000400001

58. Negri G. Diabetes melito: plantas e princípios ativos naturais hipoglicemiantes. Rev Brasil Ciên Farmac, 2005;41(2):121-142, doi 10.1590/s1516-93322005000200002. 